\title{
Origin and control of exchange-bias-like phenomenon in coupled ferromagnetic $[\mathrm{Pt} / \mathrm{Co}] / \mathrm{NiFe}$ bilayers
}

\author{
A. Bollero, ${ }^{1, *}$ V. Baltz, ${ }^{2, \dagger}$ L. D. Buda-Prejbeanu, ${ }^{2}$ B. Rodmacq,${ }^{2}$ and B. Dieny ${ }^{2}$ \\ ${ }^{1}$ IMDEA Nanoscience, Instituto Madrileño de Estudios Avanzados en Nanociencia, Campus Universidad Autónoma de Madrid, \\ 28049 Madrid, Spain \\ ${ }^{2}$ SPINTEC, UMR-8191 CNRS/CEA-INAC/UJF-Grenoble 1/Grenoble-INP, 17 rue des Martyrs, 38054 Grenoble cedex 9, France
}

(Received 13 March 2011; revised manuscript received 10 July 2011; published 20 September 2011)

\begin{abstract}
Domain formation in a $[\mathrm{Pt} / \mathrm{Co}]$ multilayer with out-of-plane anisotropy leads to a shift of the hysteresis loop of an adjacent $\mathrm{NiFe}$ thin film with in-plane anisotropy in an analogous manner to the exchange-bias phenomenon typically observed in ferromagnetic/antiferromagnetic systems. This paper shows that the loop shift can be gradually tuned through modification of the magnitude of the perpendicular anisotropy of the $[\mathrm{Pt} / \mathrm{Co}] / \mathrm{NiFe}$ system by simply varying the thickness of an underlying Pt buffer layer. Magnetometry, magnetotransport, and magnetic force microscopy experimental measurements were made. It appears that the key parameter which controls the exchange-bias effect in this system is the presence of a vortex core in between magnetic domains in the $[\mathrm{Pt} / \mathrm{Co}]$ multilayer. The magnitude of the exchange-bias is related to the ability to unpin this vortex core, which is related to the magnitude of the perpendicular anisotropy. Micromagnetic simulations illustrate the magnetic configuration responsible for the exchange-bias phenomenon in this nonstandard exchange-biased type system.
\end{abstract}

\section{INTRODUCTION}

Setting a reference direction for the spin of conduction electrons constitutes a prerequisite in most spintronic devices. ${ }^{1}$ Exchange-bias (EB), which refers to the exchange interaction between ferromagnet (FM) and antiferromagnet (AFM) layers in direct contact, is commonly used for shifting the hysteresis loop along the magnetic field axis by a quantity termed exchange-bias field, $H_{E}{ }^{2}$ To set the bias, the stack has to undergo field cooling from above the blocking temperature of the AFM bilayer. Coupling FM layers with distinct anisotropy directions has already proven to be an alternative solution in order to obtain shifted loops. ${ }^{3-8}$ By means of in-plane saturation, the formation of remanent domains in a $[\mathrm{Pt} / \mathrm{Co}]$ multilayer with out-of-plane anisotropy leads to a shift of the hysteresis loop of an adjacent $\mathrm{NiFe}$ thin film with in-plane anisotropy without the need for high-temperature anneals. ${ }^{5}$ Cain and Kryder $^{7}$ also explained this exchange-bias-like effect in a coupled $\mathrm{NiFe}-\mathrm{TbCo}(\mathrm{FM} / \mathrm{FM})$ bilayer as due to a rotation of the magnetization in the TbCo layer from in plane at the interface to perpendicular away from the interface. More recently, Yang et al. ${ }^{8}$ also observed in-plane loop shifts of a Co layer in a dual spin valve of $[\mathrm{Pd} / \mathrm{Co}] / \mathrm{Cu} / \mathrm{Co} / \mathrm{Cu} /[\mathrm{Co} / \mathrm{Pd}]$, i.e. deprived from AFM layer. They ascribed this exchange-bias-like behavior as a result of the magnetostatic interaction between the net in-plane $\mathrm{Co} / \mathrm{Pd}$ remanent moments and the Co moments. They also suggested the possible presence of flux closure caps with unequal size in their system formed after in-plane saturation contributing to the in-plane stray field responsible for the exchange-bias. The asymmetry of the multilayer magnetic configuration is of fundamental importance in order to achieve a loop shift for the $\mathrm{NiFe}$ in the $[\mathrm{Pt} / \mathrm{Co}] / \mathrm{NiFe}$ system. ${ }^{9}$ An initial demagnetized state for the $[\mathrm{Pt} / \mathrm{Co}]$ multilayer would result in no loop shift as a consequence of the formation of equally sized closure domains. A previous study on $[\mathrm{Pt} / \mathrm{Co}] / \mathrm{NiFe}$ showed the possibility of obtaining a threefold increase of $H_{E}$ in a patterned system consisting of parallel long stripes as compared to a continuous film and following in-plane saturation of the nanostructures. ${ }^{10}$
Controlling the geometry (size and shape) and the pinning of the above-mentioned domains and domain walls would mean being able to tune the magnitude of the hysteresis loop shift for coupled ferromagnetic layers with distinct magnetic anisotropy. That is the purpose of this paper, which shows that the hysteresis loop shift of a NiFe layer with in-plane anisotropy in contact with a $[\mathrm{Pt} / \mathrm{Co}]$ multilayer with out-of-plane anisotropy in a maze domain configuration can be tuned by varying the thickness of an underlying Pt buffer layer. The influence of the Pt buffer thickness on the anisotropy constant and structural properties was measured. The results can be consistently explained by the presence of vortex cores in between the magnetic domains in the $[\mathrm{Pt} / \mathrm{Co}]$ multilayer and their ability of being more or less pinned depending on the magnitude of the perpendicular anisotropy, which itself is varied with the thickness of the buffer layer.

\section{EXPERIMENTAL PROCEDURE}

Sheet films with compositions $\mathrm{Pt}\left(\mathrm{t}_{\mathrm{Pt}}\right) /\left[\mathrm{Co}_{0.6 \mathrm{~nm}} /\right.$ $\left.\mathrm{Pt}_{1.8 \mathrm{~nm}}\right]_{5} / \mathrm{Co}_{0.2 \mathrm{~nm}} / \mathrm{NiFe}_{3 \mathrm{~nm}} / \mathrm{Cu}_{2 \mathrm{~nm}} / \mathrm{Pt}_{2 \mathrm{~nm}}$ and $\mathrm{Pt}\left(\mathrm{t}_{\mathrm{Pt}}\right) /[$ $\left.\mathrm{Co}_{0.6 \mathrm{~nm}} / \mathrm{Pt}_{1.8 \mathrm{~nm}}\right]_{5}$ were fabricated onto thermally oxidized silicon substrates. The number of $\mathrm{Co} / \mathrm{Pt}$ repeats and the $\mathrm{NiFe}$ thickness were chosen on the basis of previous results, ${ }^{6}$ where both parameters were optimized in order to increase the exchange-bias-like phenomenon. The Pt buffer thickness ranges from 0 to $25 \mathrm{~nm}$. Deposition was performed at room temperature by means of dc-magnetron sputtering with an Ar plasma under a pressure of $2.5 \times 10^{-3}$ mbar and following a base pressure of $4.9 \times 10^{-8}$ mbar. In order to ease the reading, the following notations will be used throughout the manuscript: $[\mathrm{Pt} / \mathrm{Co}] / \mathrm{NiFe}$ for $\mathrm{Pt}\left(\mathrm{t}_{\mathrm{Pt}}\right) /\left[\mathrm{Co}_{0.6 \mathrm{~nm}} / \mathrm{Pt}_{1.8 \mathrm{~nm}}\right]_{5} / \mathrm{Co}_{0.2 \mathrm{~nm}} / \mathrm{NiFe}_{3 \mathrm{~nm}} / \mathrm{Cu}_{2 \mathrm{~nm}} / \mathrm{Pt}_{2 \mathrm{~nm}}$ and $[\mathrm{Pt} / \mathrm{Co}]$ for $\mathrm{Pt}\left(\mathrm{t}_{\mathrm{Pt}}\right) /\left[\mathrm{Co}_{0.6 \mathrm{~nm}} / \mathrm{Pt}_{1.8 \mathrm{~nm}}\right]_{5}$.

Hysteresis loops were measured at room temperature along the in-plane direction by vibrating sample magnetometry (VSM). To set the loop shift, an in-plane field of $15 \mathrm{kOe}$ was applied large enough to in-plane saturate both the $[\mathrm{Pt} / \mathrm{Co}]$ 

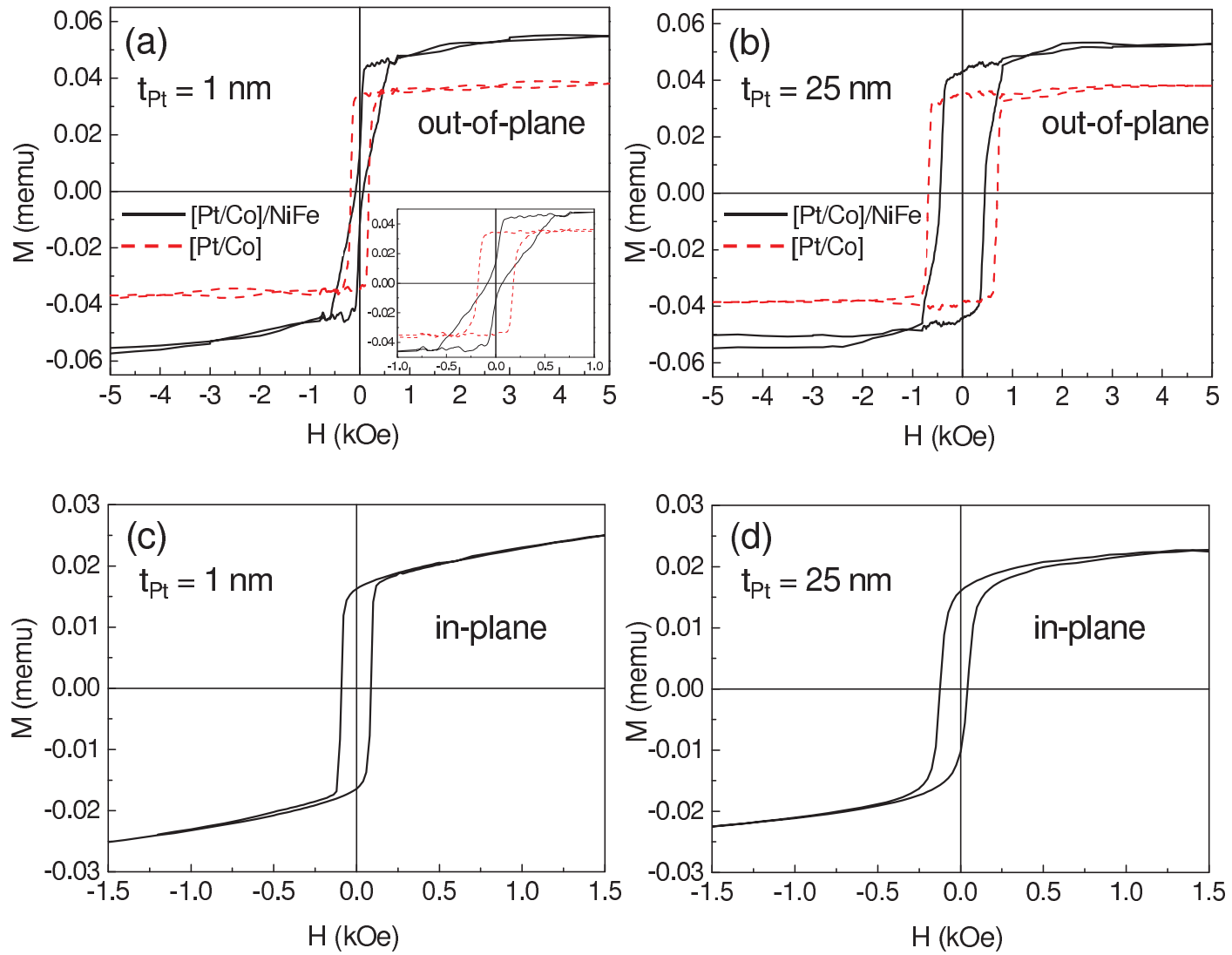

FIG. 1. (Color online) Out-of-plane hysteresis loops measured by VSM for $\mathrm{Pt}\left(\mathrm{t}_{\mathrm{Pt}}\right) /\left[\mathrm{Co}_{0.6 \mathrm{~nm}} / \mathrm{Pt}_{1.8 \mathrm{~nm}}\right]_{5} / \mathrm{Co}_{0.2 \mathrm{~nm}} / \mathrm{NiFe}_{3 \mathrm{~nm}} / \mathrm{Cu}_{2 \mathrm{~nm}} / \mathrm{Pt}_{2 \mathrm{~nm}}$ (continuous line) and $\mathrm{Pt}\left(\mathrm{t}_{\mathrm{Pt}}\right) /\left[\mathrm{Co}_{0.6 \mathrm{~nm}} / \mathrm{Pt}_{1.8 \mathrm{~nm}}\right]_{5}$ (dashed line) with (a) $t_{\mathrm{Pt}}=1 \mathrm{~nm}$ and (b) $t_{\mathrm{Pt}}=25 \mathrm{~nm}$. Inset in (a): zoom of the out-of-plane hysteresis loops for $[\mathrm{Pt} / \mathrm{Co}] / \mathrm{NiFe}$ and $[\mathrm{Pt} / \mathrm{Co}]$ with $t_{\mathrm{Pt}}=1 \mathrm{~nm}$. In-plane hysteresis loops for $[\mathrm{Pt} / \mathrm{Co}] / \mathrm{NiFe}$ with $(\mathrm{c}) t_{\mathrm{Pt}}=1 \mathrm{~nm}$ and $(\mathrm{d}) t_{\mathrm{Pt}}=25 \mathrm{~nm}$ after applying an in-plane field of $15 \mathrm{kOe}$ and subsequent measurement of the in-plane hysteresis loop with fields ranging between $\pm 1.5 \mathrm{kOe}$.

multilayer with out-of-plane anisotropy and the NiFe thin film with in-plane anisotropy. The in-plane hysteresis loop was subsequently measured with a maximum field of $1.5 \mathrm{kOe}$, i.e. large enough to saturate the $\mathrm{NiFe}$ but insufficient to saturate the $[\mathrm{Pt} / \mathrm{Co}]$ multilayer. Along the out-of-plane direction, the measurements were obtained at room temperature by VSM and by extraordinary Hall effect (EHE). This latter method is particularly sensitive to the out-of-plane component of the magnetization ${ }^{11,12}$ and was also used to determine the anisotropy constant of the $[\mathrm{Pt} / \mathrm{Co}]$ multilayer. For that purpose, following saturation of the films along the out-of-plane direction, the application of an increasing in-plane magnetic field progressively reorients the magnetization in-plane, which manifests itself by a decrease of the EHE signal. ${ }^{13}$

Structural data were extracted from large angle $\mathrm{x}$-ray diffractometry (XRD). The $K_{\alpha}$ emission of a Cu source with a wavelength of $0.15418 \mathrm{~nm}$ was used. Specular curves $(\theta-2 \theta)$ as well as rocking curves $(2 \theta-\omega)$ were measured.

Atomic force microscopy showed a root-mean-square (RMS) roughness of about $0.6 \pm 0.1 \mathrm{~nm}$ for bare [Pt/Co] multilayers independently of the thickness of the Pt buffer layer, in good agreement with results reported for similar $[\mathrm{Pt} / \mathrm{Co}]$ systems with varying $t_{\mathrm{Pt}} .{ }^{14}$ The magnetic domains of the samples were imaged at remanence by magnetic force microscopy (MFM) after ex-situ in-plane saturation under a $15 \mathrm{kOe}$ magnetic field. Micromagnetic simulations were performed following the same experimental field history.

\section{RESULTS AND DISCUSSION}

Typical hysteresis loops measured by VSM along the out-of-plane direction are shown in Figs. 1(a) and 1(b) for coupled $[\mathrm{Pt} / \mathrm{Co}] / \mathrm{NiFe}$ stacks and $[\mathrm{Pt} / \mathrm{Co}]$ multilayers with $\mathrm{Pt}$ buffer layer thicknesses of 1 and $25 \mathrm{~nm}$, respectively. Note that all samples exhibit the same structure, which allows comparing the values of magnetic moment obtained by VSM. From Fig. 1, one observes looking separately at $[\mathrm{Pt} / \mathrm{Co}]$ and $[\mathrm{Pt} / \mathrm{Co}] / \mathrm{NiFe}$ that the saturation magnetic moment is independent of the buffer thickness. A direct comparison between the loops of both systems shows that the effects of adding a NiFe layer on top of the $[\mathrm{Pt} / \mathrm{Co}]$ on the corresponding out-of-plane hysteresis loops are yet noticeable. An enhanced remanent magnetization is obtained by adding NiFe for sufficiently thick Pt buffer layer $\left(t_{\mathrm{Pt}}>1 \mathrm{~nm}\right)$, which is indicative of a net out-of-plane contribution of the NiFe layer, as will be further discussed. From Fig. 1, one also measures a saturation moment of the $[\mathrm{Pt} / \mathrm{Co}]$ equal to 0.037 memu and a saturation moment of the $\mathrm{NiFe}$ equal to $0.017 \mathrm{memu}$ (this value is the difference between the moment of the $[\mathrm{Pt} / \mathrm{Co}] / \mathrm{NiFe}$ system at saturation, continuous line in Fig. 1, and that of the [Pt/Co] multilayer, dashed line in Fig. 1). The ratio between the two agrees well with our 
nominal thicknesses and standard saturation magnetizations for $\mathrm{Co}$ and NiFe. Additionally, and independently of the buffer thickness, the presence of NiFe results in a clear alteration of the out-of-plane hysteresis loop shape and in particular its squareness, meaning that the susceptibility has been lowered. This can reasonably be ascribed to a reduction of the effective out-of-plane anisotropy since NiFe shows in-plane anisotropy. The susceptibility is roughly proportional to the square root of the effective out-of-plane anisotropy. ${ }^{15}$ As a consequence of a reduction of the effective out-of-plane anisotropy, a reduction of the out-of-plane coercive field, $H_{c}$ (Fig. 1) is observed. In particular, the shape of the out-of-plane hysteresis loop for $[\mathrm{Pt} / \mathrm{Co}] / \mathrm{NiFe}$ with $t_{\mathrm{Pt}}=0 \mathrm{~nm}$ (not shown) and $t_{\mathrm{Pt}}=1 \mathrm{~nm}$ [inset in Fig. 1(a)] is similar to that obtained for $[\mathrm{Pt} / \mathrm{Co}]$ systems with a large number of $\mathrm{Co} / \mathrm{Pt}$ bilayers as, for example, to that measured by Davies et al. ${ }^{16}$ for a $\mathrm{Pt}_{20 \mathrm{~nm}} /\left[\mathrm{Co}_{0.4 \mathrm{~nm}} / \mathrm{Pt}_{0.7 \mathrm{~nm}}\right]_{50}$ system. These shapes result from a balance between a gain in magnetostatic energy associated with up and down domain formation and a cost in domain wall and Zeeman energy. As a consequence, a change of sign of the nucleation field and a correlative reduced remanence to saturation magnetization ratio, $M_{r} / M_{s}$, were measured when reducing the Pt buffer layer thickness [Figs. 1(a) and 1(b)]. Values of $M_{r} / M_{s}$ equal to 0.07 , 0.33 , and $0.82-0.88$ were obtained for $t_{\mathrm{Pt}}$ respectively equal to 0,1 , and $3-25 \mathrm{~nm}$ in the $[\mathrm{Pt} / \mathrm{Co}] / \mathrm{NiFe}$ system. These loop shapes contrast with the sharp transitions obtained for the bare [Pt/Co] multilayers [Figs. 1(a) and 1(b)], which originate from magnetization reversal due to nucleation and subsequent fast propagation of reverse domains. An increase of the Pt buffer thickness leads to an increased squareness of the out-of-plane hysteresis loop for the coupled $[\mathrm{Pt} / \mathrm{Co}] / \mathrm{NiFe}$ stacks and enhanced out-of-plane $H_{c}$ values for both [Pt/Co]/NiFe and [Pt/Co] [see Figs. 1(a) and 1(b) and Fig. 2, respectively]. As will be confirmed later, the fact that the loops get less slanted (larger susceptibility) when $t_{\mathrm{Pt}}$ is increased is likely due to an increase of the out-of-plane anisotropy. ${ }^{15}$

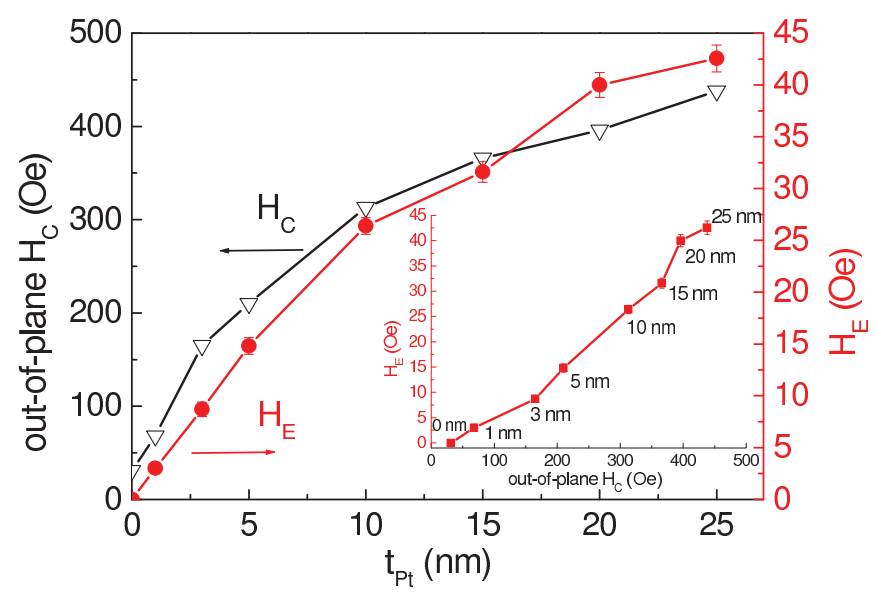

FIG. 2. (Color online) Evolution of the loop shift $H_{E}$ (full symbols) and the out-of-plane coercivity $H_{c}$ (empty symbols) deduced from in-plane and out-of-plane hysteresis loops (as those shown in Fig. 1), respectively, with the thickness of the Pt buffer layer, $t_{\mathrm{Pt}}$, for $[\mathrm{Pt} / \mathrm{Co}] / \mathrm{NiFe}$. Inset: $H_{E}$ vs out-of-plane $H_{c}$ for the different $t_{\mathrm{Pt}}$ values (specified next to each symbol).
Typical in-plane hysteresis loops measured by VSM for $[\mathrm{Pt} / \mathrm{Co}] / \mathrm{NiFe}$ with $t_{\mathrm{Pt}}=1 \mathrm{~nm}$ and $t_{\mathrm{Pt}}=25 \mathrm{~nm}$ with fields ranging between $\pm 1.5 \mathrm{kOe}$, after applying an in-plane field of $15 \mathrm{kOe}$, are shown in Figs. 1(c) and 1(d), respectively. Note that, for these in-plane hysteresis loops, the curves look squarer for thinner $t_{\mathrm{Pt}}$. This is also consistent with the above-mentioned decreased perpendicular anisotropy with decreasing $t_{\mathrm{Pt}}$. Additionally, an enhanced loop shift, $H_{E}$, is obtained when increasing $t_{\mathrm{Pt}}$. Figure 2 shows a comparison of the $t_{\mathrm{Pt}}$ dependence of both out-of-plane coercivity $H_{c}$ and loop shift $H_{E}$. Both evolutions are very similar, indicating a correlation between the magnetic anisotropy in the $[\mathrm{Pt} / \mathrm{Co}]$ multilayer and the shift in the in-plane hysteresis loop of the NiFe layer. The similarity is such that a plot of $H_{E}$ vs out-of-plane $H_{c}$ results in a practically straight line (see inset of Fig. 2). The fact that the in-plane hysteresis loop for $[\mathrm{Pt} / \mathrm{Co}] / \mathrm{NiFe}$ with no Pt buffer layer $\left(t_{\mathrm{Pt}}=0 \mathrm{~nm}\right)$ does not virtually show any loop shift and displays a significantly reduced out-of-plane $H_{c}$ might be a direct consequence of a detrimental crystalline texture of this multilayered system. The presence of a very thin Pt buffer layer of $1 \mathrm{~nm}$ is sufficient to yield a loop shift of 3 Oe, which increases up to $\sim 45$ Oe for $t_{\mathrm{Pt}}=25 \mathrm{~nm}$, while $H_{c}$ increases from 68 to $438 \mathrm{Oe}$, respectively. XRD analysis of the reference $[\mathrm{Pt} / \mathrm{Co}$ ] system with $t_{\mathrm{Pt}}=0-25 \mathrm{~nm}$ was carried out in order to get further insight into the influence of the thickness of the Pt buffer layer on the structural quality of the multilayer.

XRD analysis shows a strong $\langle 111\rangle$ orientation for all [Pt/Co] films with $t_{\mathrm{Pt}}>1 \mathrm{~nm}$. [Pt/Co] films with no $\mathrm{Pt}$ buffer layer or containing a very thin one (1 nm thick) also exhibit a preferred but significantly weaker $\langle 111\rangle$ orientation. No additional structural differences were observed between the $[\mathrm{Pt} / \mathrm{Co}]$ films of this study. However, the origin of the large enhancement of the $\operatorname{Pt}(111)$ orientation with increasing $t_{\mathrm{Pt}}$ cannot be easily determined from standard $(\theta-2 \theta)$ specular measurements. It could be ascribed to an effective improved texture of the system but also to a stronger signal resulting from the increasing Pt buffer thickness. Figure 3 shows the



FIG. 3. Evolution with $t_{\mathrm{Pt}}$ of FWHM determined from the XRD rocking curves (inset) measured for the (111) peak of $[\mathrm{Pt} / \mathrm{Co}]$ with various buffer thicknesses, $t_{\mathrm{Pt}}$ 


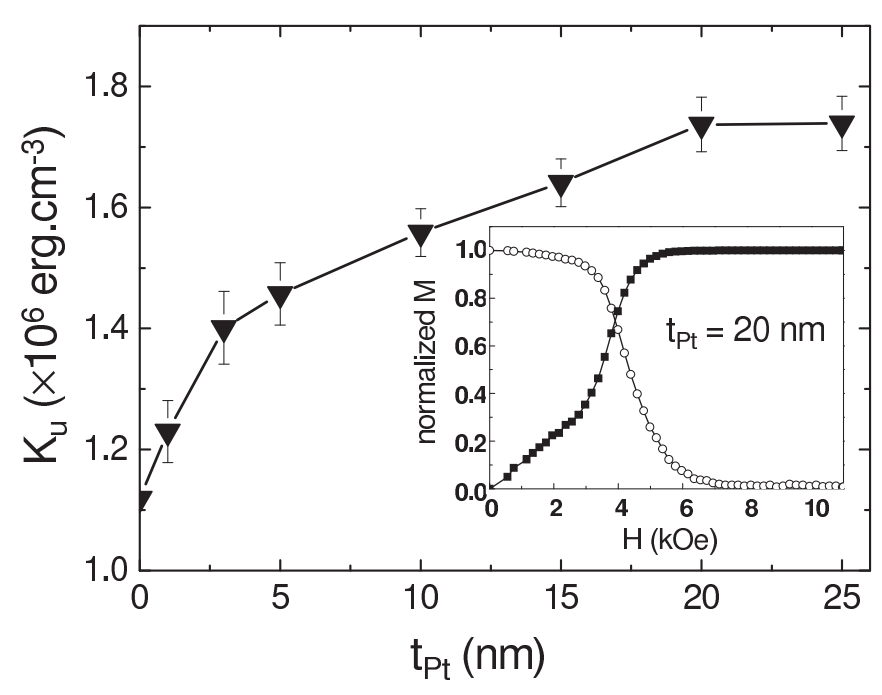

FIG. 4. Evolution with $t_{\mathrm{Pt}}$ of the uniaxial anisotropy, $K_{u}$, for [Pt/Co]. Inset: Determination of the anisotropy field for $t_{\mathrm{Pt}}=20 \mathrm{~nm}$ where empty symbols denote the evolution of the $M_{z}$ component of the magnetization measured by EHE with an in-plane applied field; full symbols indicate the deduced evolution of the in-plane component of the magnetization $M_{x}\left(M_{x}^{2}+M_{z}^{2}=1\right)$ with the applied field.

$(2 \theta-\omega)$ rocking curves and the evolution with $t_{\mathrm{Pt}}$ of the full width at half maximum (FWHM) values for the [Pt/Co] films. A decrease in FWHM can be observed with increasing $t_{\mathrm{Pt}}$ up to $20 \mathrm{~nm}$. No significant changes are observed between FWHM values for $t_{\mathrm{Pt}}=20$ and $25 \mathrm{~nm}$. The evolution with increasing $t_{\mathrm{Pt}}$ of the FWHM of the rocking curves might be indicative of an enhanced (111) texture of the [Pt/Co] multilayer, ${ }^{17,18}$ although FWHM values may be influenced by different microstructural parameters. ${ }^{19}$ The correlation between the apparent improved structural quality of the system with increasing $t_{\mathrm{Pt}}$ and the perpendicular magnetic anisotropy was studied by measuring the anisotropy constant of the bare $[\mathrm{Pt} / \mathrm{Co}]$ multilayer vs thickness of the buffer layer by EHE.

The evolution with $t_{\mathrm{Pt}}$ of the uniaxial anisotropy, $K_{u}$, for $\operatorname{Pt}\left(t_{\mathrm{Pt}}\right) /\left[\mathrm{Co}_{0.6 \mathrm{~nm}} / \mathrm{Pt}_{1.8 \mathrm{~nm}}\right]_{5}$ is shown in Fig. 4. A typical raw data (for the case $t_{\mathrm{Pt}}=20 \mathrm{~nm}$ ) from which the effective anisotropy is extracted is shown in the inset of Fig. $4 .{ }^{13} \mathrm{It}$ is possible to see that an increased thickness of the Pt buffer layer results in an enhanced $K_{u}$ with a maximum value of $1.74 \times 10^{6}$ erg.cm ${ }^{-3}$ for $t_{\mathrm{Pt}}=25 \mathrm{~nm}$. Here, $K_{u}$ remains practically unchanged for a Pt buffer thickness above $20 \mathrm{~nm}$ $\left(K_{u}=1.73 \times 10^{6}\right.$ erg.cm $\left.{ }^{-3}\right)$. This agrees with the enhanced structural quality of [Pt/Co] with increasing $t_{\mathrm{Pt}}$ suggested by XRD (Fig. 3) and the absence of further improvement when continuing to increase $t_{\mathrm{Pt}}$ above $20 \mathrm{~nm}$. The large enhancement of the $\mathrm{Pt}(111)$ orientation and $[\mathrm{Pt} / \mathrm{Co}](111)$ texture associated with increasing the thickness of the Pt buffer is likely at the origin of the increased perpendicular magnetic anisotropy of the $[\mathrm{Pt} / \mathrm{Co}]$ multilayer. This observation is in agreement with previous literature. ${ }^{20}$ The reduced perpendicular anisotropy of [Pt/Co] obtained for low $t_{\mathrm{Pt}}$ values (Fig. 4) explains the more slanted out-of-plane hysteresis loops (and significantly decreased $M_{r} / M_{s}$ ratios) when adding a NiFe layer (with in-plane anisotropy) on top [see Fig. 1(a) for $t_{\mathrm{Pt}}=1 \mathrm{~nm}$ ], by comparison with the enhanced perpendicular anisotropy obtained with increasing $t_{\mathrm{Pt}}$, which leads to squarer hysteresis loops and increased $M_{r} / M_{s}$ values [see Fig. 1(b) for $t_{\mathrm{Pt}}=$ $25 \mathrm{~nm}]$.

In order to compare the magnetic domain structures of $[\mathrm{Pt} / \mathrm{Co}]$ and $[\mathrm{Pt} / \mathrm{Co}] / \mathrm{NiFe}$ for various thicknesses of the Pt buffer layer and to check for a potential link between domain structure and $H_{E}$, MFM images were taken at remanence after in-plane saturation with $15 \mathrm{kOe}$ (Fig. 5). The images corresponding to the $[\mathrm{Pt} / \mathrm{Co}] / \mathrm{NiFe}$ mainly show the magnetic contrast stemming from the stripe domains in the $[\mathrm{Pt} / \mathrm{Co}]$ multilayer. It must be noted that these images do not correspond to an equilibrium configuration, as they result from saturation of the whole multilayered system by applying a large in-plane magnetic field $(15 \mathrm{kOe})$ followed by subsequent field suppression to achieve the visualized remanent state.

The mean magnetic domain sizes were deduced from a log normal fit of the Fourier transform of the image (Fig. 5). The Fourier transform is centered on the mean wavelength, which is twice the mean domain size. Such a typical curve and fit are shown in Fig. 6 for $[\mathrm{Pt} / \mathrm{Co}] / \mathrm{NiFe}$ with $t_{\mathrm{Pt}}=$ $20 \mathrm{~nm}$. The dependence with $t_{\mathrm{Pt}}$ of the mean magnetic domain sizes is shown in Fig. 7. For $t_{\mathrm{Pt}} \leqslant 3 \mathrm{~nm}$, the mean magnetic domain size increases for both $[\mathrm{Pt} / \mathrm{Co}]$ and $[\mathrm{Pt} / \mathrm{Co}] / \mathrm{NiFe}$ systems with increasing $t_{\mathrm{Pt}}$. This effect might be due to the poorer crystalline texture of the multilayer observed for an excessively thin buffer layer. For a sufficiently thick Pt buffer layer $\left(t_{\mathrm{Pt}}>3 \mathrm{~nm}\right)$ and for both $[\mathrm{Pt} / \mathrm{Co}]$ and $[\mathrm{Pt} / \mathrm{Co}] / \mathrm{NiFe}$ systems - importantly, within error bars-the domain sizes seem to be approximately independent of $t_{\mathrm{Pt}}$. The domain size is yet known to increase exponentially with the square root of the exchange times the anisotropy. The fact that the domain size remains practically constant for $t_{\mathrm{Pt}}>3 \mathrm{~nm}$ despite increasing values of anisotropy suggests that the exchange stiffness would take a reduced value with increasing $t_{\mathrm{Pt}}$. This would be in agreement with the observed shift of the (111) peak of $[\mathrm{Pt} / \mathrm{Co}]$ to lower angles in the XRD patterns (not shown) with increasing the Pt buffer layer thickness. One can also note that for all considered Pt thicknesses, the addition of a $\mathrm{NiFe}$ layer on top of $[\mathrm{Pt} / \mathrm{Co}]$ results in a reduction of the mean domain size (Fig. 7) by comparison with the bare [Pt/Co] multilayer. This is a consequence of the easy-plane contribution from the $\mathrm{NiFe}$, which acts as a guide to the magnetostatic flux. The addition of the NiFe is equivalent to significantly reducing the effective anisotropy of the system, and this effect will be more pronounced for those [Pt/Co] systems with a weaker perpendicular anisotropy (thinner $\mathrm{Pt}$ buffer layer). Related to this, it is interesting to note that magnetic domains (Fig. 5) are elongated in the direction parallel to the saturation direction for $[\mathrm{Pt} / \mathrm{Co}] / \mathrm{NiFe}$ with a thin Pt buffer layer $\left(t_{\mathrm{Pt}}<5 \mathrm{~nm}\right)$. To verify this tendency, two-dimensional fast Fourier transformations (2D FFTs) were obtained from the MFM images of Fig. $5 .^{21}$ Inset of each figure shows a detailed image of the corresponding intensity plots of the 2D FFT images. Anisotropy in the FFT is observed for $[\mathrm{Pt} / \mathrm{Co}] / \mathrm{NiFe}$ containing a buffer layer with a thickness below $5 \mathrm{~nm}$ as a consequence of the stripe-like magnetic domains oriented parallel to the saturating applied field (most clearly seen for $t_{\mathrm{Pt}}=0 \mathrm{~nm}$ ). However, an increased thickness of the $\mathrm{Pt}$ 

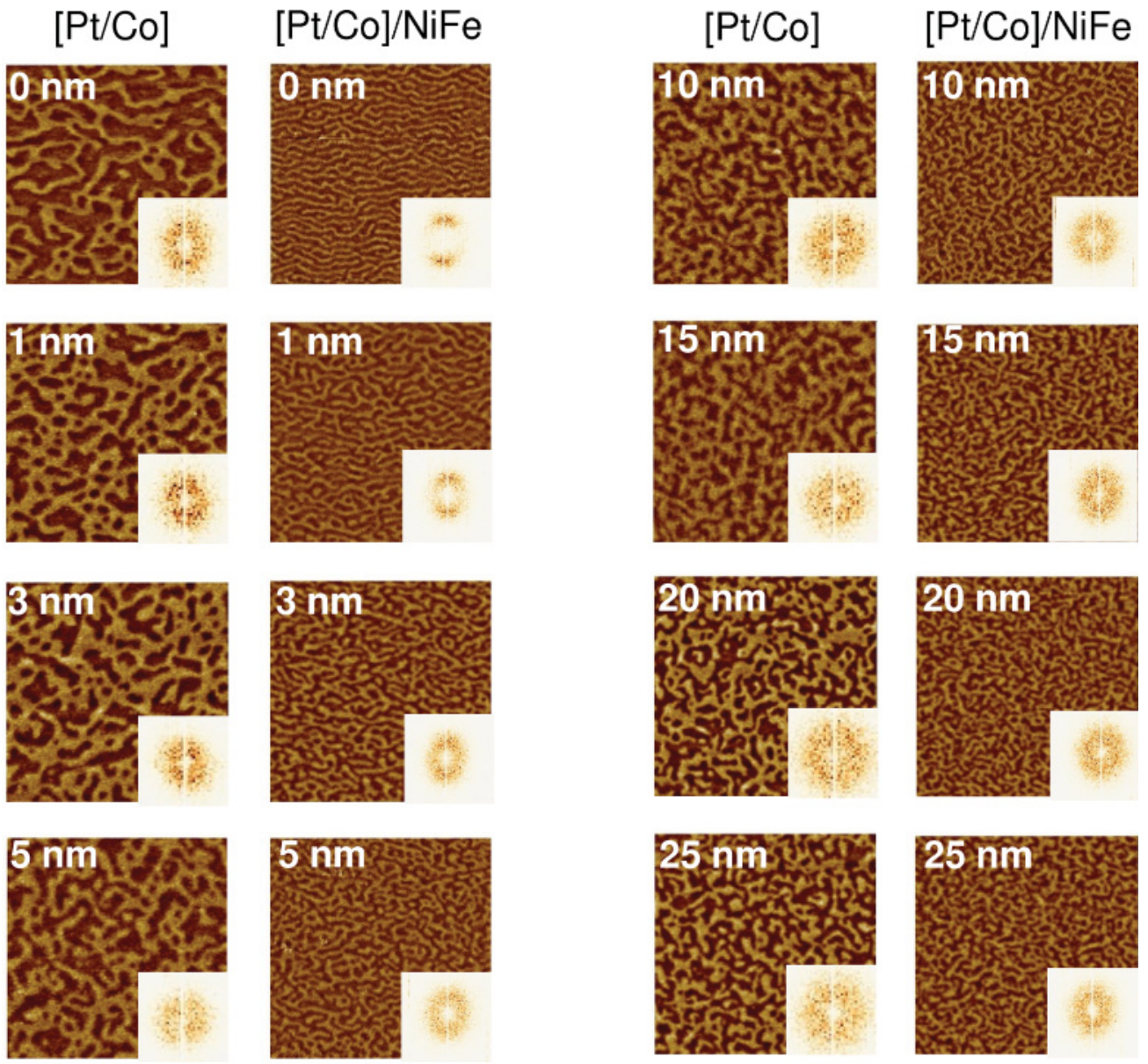

\section{$2 \mu \mathrm{m}$}

\section{$2 \mu \mathrm{m}$}

FIG. 5. (Color online) MFM images for $[\mathrm{Pt} / \mathrm{Co}]$ and $[\mathrm{Pt} / \mathrm{Co}] / \mathrm{NiFe}$ with various buffer thicknesses, $t_{\mathrm{Pt}}$, at remanence after in-plane saturation with $15 \mathrm{kOe}$. Scale bar for MFM images: $2 \mu \mathrm{m}$. Insets show corresponding details of MFM images after two-dimensional Fourier transform with scaling $\times 1$ for $[\mathrm{Pt} / \mathrm{Co}]$ and $\times 0.5$ for $[\mathrm{Pt} / \mathrm{Co}] / \mathrm{NiFe}$.

buffer layer results in isotropic FFT with no directionality in the shape because of the random orientation of the magnetic domains in a maze-like configuration. In fact the labyrinth shape of the domains in the $[\mathrm{Pt} / \mathrm{Co}] / \mathrm{NiFe}$ system with $t_{\mathrm{Pt}}>$ $5 \mathrm{~nm}$ is very similar to that of the corresponding [Pt/Co] multilayer without $\mathrm{NiFe}$. The observation that the $2 \mathrm{D}$ spectra are larger with the $\mathrm{NiFe}$ than without (considering that FFT images for $[\mathrm{Pt} / \mathrm{Co}] / \mathrm{NiFe}$ shown in Fig. 5 are reduced to half their original size for better comparison with those for [Pt/Co], which have not been reduced in size) agrees with the fact that the domains are smaller (since the domain size is inversely proportional to the size of the $2 \mathrm{D}$ spectrum) for $[\mathrm{Pt} / \mathrm{Co}$ containing $\mathrm{NiFe}$ on top. Additionally, the fact that the $2 \mathrm{D}$ spectra for both systems become smaller with increasing $t_{\mathrm{Pt}}$ for $t_{\mathrm{Pt}} \leqslant 3 \mathrm{~nm}$ agrees with the fact that the domain sizes become larger in this thickness range as shown in Fig. 7, with practically no size variation for larger Pt thicknesses.

The use of a simple model along with illustrating micromagnetic simulations can give further insight into the link between domain structure and $H_{E}$. If one considers simple
Bloch walls between the domains in the $[\mathrm{Pt} / \mathrm{Co}]$ multilayer, the core of the Bloch wall is pointing perpendicularly to the wall plane. Therefore, and in view of the previously discussed results, the application of a strong magnetic field of $15 \mathrm{kOe}$ followed by subsequent removal will probably result in all the cores of the wall tending to orient within the same direction. This core certainly leads to hysteresis loop shift when cycling the magnetization of the sole NiFe. It is also likely that the larger the anisotropy, the harder it is to move this core, and the larger the amplitude of the loop shift. This mechanism would also be consistent with the fact that the loop shift can be directly related to the out-of-plane coercive field as plotted in the inset of Fig. 2. In Refs. 5 and 6, it was proposed that the loop shift along the field axis measured for the $[\mathrm{Pt} / \mathrm{Co}] / \mathrm{NiFe}$ system originates from uncompensated moments stemming from unequally oriented closure domains at the $[\mathrm{Pt} / \mathrm{Co}] / \mathrm{NiFe}$ interface, created after application of a small in-plane field, in analogy to the uncompensated spins in the AFM in conventional FM-AFM exchange-biased bilayers. However, this description lacked the important implications on the exchange-bias phenomenon following the formation, 


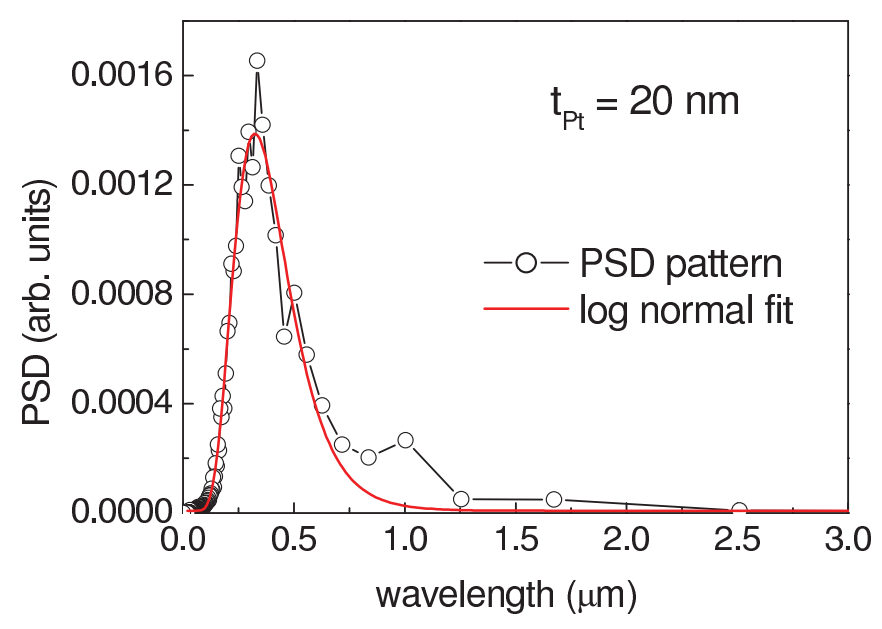

FIG. 6. (Color online) Power spectral densities (PSD) pattern of MFM image from Fig. 5 for $[\mathrm{Pt} / \mathrm{Co}] / \mathrm{NiFe}$ with $t_{\mathrm{Pt}}=20 \mathrm{~nm}$ (empty symbols). Continuous (red) line is a log normal fit of the corresponding Fourier transform.

the asymmetry, and the effective stability of vortices in the walls separating upwards and downwards domains in the $[\mathrm{Pt} / \mathrm{Co}]$ multilayer. In this paper, where a nonequilibrium state is obtained after in-plane saturation of the whole system, the tiny loop shift observed for the $[\mathrm{Pt} / \mathrm{Co}] / \mathrm{NiFe}$ system with an excessively thin buffer layer, i.e. reduced perpendicular anisotropy unable of pinning effectively the vortices against magnetization reversal, shows the importance of both factors.

Micromagnetic simulations ${ }^{22}$ were performed following the same experimental field history. In the simulations, the domain pattern was assumed to be periodic, thus rending the problem two dimensional. The $[\mathrm{Pt} / \mathrm{Co}]$ multilayer was considered as a single ferromagnetic layer having an exchange constant $A_{e x}=1.0 \times 10^{-7} \mathrm{erg} \cdot \mathrm{cm}^{-1}$. The out-of-plane

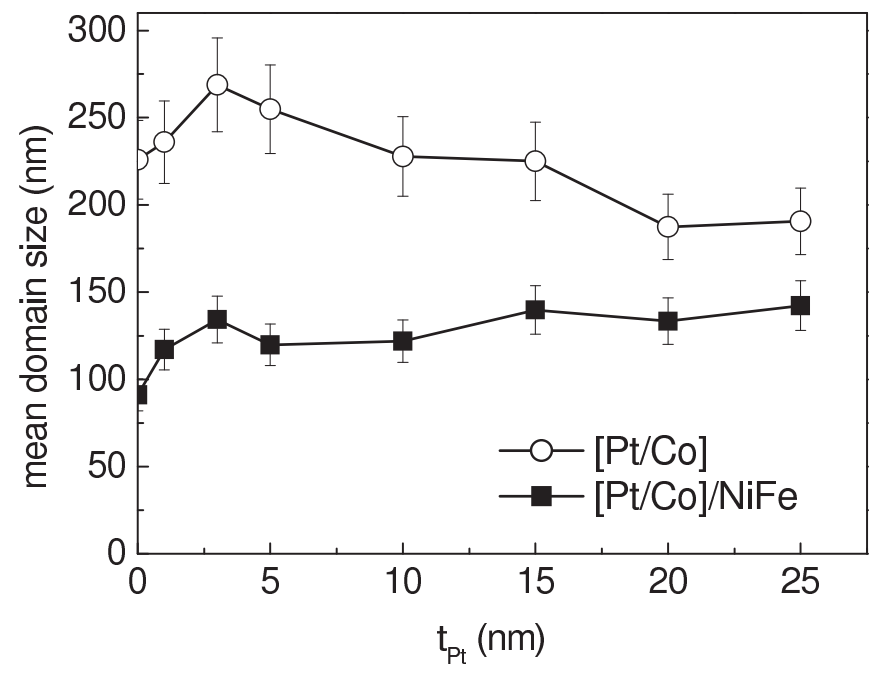

FIG. 7. Evolution of the mean magnetic domain size with $t_{\mathrm{Pt}}$ for $[\mathrm{Pt} / \mathrm{Co}] / \mathrm{NiFe}$ (full symbols) and $[\mathrm{Pt} / \mathrm{Co}]$ (empty symbols) determined from the MFM images of Fig. 5. uniaxial anisotropy constant was taken from the experimental results in Fig. 4. The saturation magnetization was $M_{s}=350 \mathrm{emu} \cdot \mathrm{cm}^{-3}$. These values are comparable to values reported in the literature. ${ }^{23,24}$ Standard values $A_{e x}=1.3 \times$ $10^{-6} \mathrm{erg} \cdot \mathrm{cm}^{-1}$ and $M_{s}=800 \mathrm{emu} \cdot \mathrm{cm}^{-3}$ were considered for the NiFe layer. No magnetic anisotropy was considered in NiFe. The mesh size was $0.5 \times 0.2 \mathrm{~nm}^{2}$, smaller than the magnetic characteristic lengths of the system (exchange length and domain wall width). A typical cross-sectional view from the simulation is given in Fig. 8. The domain structure in the $[\mathrm{Pt} / \mathrm{Co}]$ part of the $[\mathrm{Pt} / \mathrm{Co}] / \mathrm{NiFe}$ stack after in-plane saturation results in a maze of alternating upwards and downwards magnetized domains separated by the walls. The limiting factor to take into account is the two-dimensional character of the simulations, which results in an equilibrium configuration consisting of ideal parallel stripes; this is obviously not the case for the system under study, where a labyrinth pattern is observed (see MFM images in Fig. 5) at remanence after in-plane saturation of the whole multilayer. As already reported in Ref. 6, the simulations show the presence of Nél-type flux closure caps in both $[\mathrm{Pt} / \mathrm{Co}]$ and $[\mathrm{Pt} / \mathrm{Co}] / \mathrm{NiFe}$ systems on top of Bloch walls. These closure caps originate from a magnetization circulation that takes place at the top of the $[\mathrm{Pt} / \mathrm{Co}]$ multilayer (and $[\mathrm{Pt} / \mathrm{Co}] / \mathrm{NiFe}$ interface) and at the bottom surface after inplane saturation. This magnetic configuration results in Blochlike walls located in the film center. In Fig. 8(b), the color code is associated with the $z$ component of the magnetization, which corresponds to the direction of initial application of the in-plane saturating field. Interestingly, the magnetization in the core of the vortex is pointing in the $z$ direction, with a circulation which determines the magnetization direction in the closure cap and, consequently, in the coupled NiFe layer. Additional information on the magnetic configuration can be extracted by analyzing the contribution of each component of the magnetization $\left(M_{x}, M_{y}\right.$, and $\left.M_{z}\right)$ at remanence after application of an in-plane saturating field [along the $z$ axis, as shown in Fig. 8(c)]. The magnetic domains in $[\mathrm{Pt} / \mathrm{Co}]$ are alternatively oriented upwards and downwards with values $M_{x}=M_{z}=0$ and $M_{y}=1$ (up) or $M_{y}=-1$ (down) prior to the beginning of tilt close to the $\mathrm{Pt} / \mathrm{Co}-\mathrm{NiFe}$ interface. The closure cap is therefore contained in both $\mathrm{Pt} / \mathrm{Co}$ and $\mathrm{NiFe}$ bilayers extending through the interface. This results in closure of the domains in a configuration where the magnetic moments follow a three-dimensional wavelike evolution, as illustrated in Fig. 8(a). As an important conclusion from this result is the inducement of an out-of-plane ( $y$ axis) contribution to the magnetization of the NiFe layer evolving from positive to negative when going from upward to downward domains in the $\mathrm{Pt} / \mathrm{Co}$ multilayer. This proves an extension of the $\mathrm{Pt} / \mathrm{Co}$ magnetic domains through the NiFe layer, and it is in good agreement with the out-of-plane VSM measurements [Fig. 1(b)] which showed an enhanced remanent magnetization when adding $\mathrm{NiFe}$. The net $\mathrm{NiFe}$ magnetization is oriented along the $z$ direction at zero field, and the resulting exchangebias field is also oriented in the $z$ direction. Therefore, it appears that the exchange-bias arises from the exchange coupling between the magnetization of the top NiFe layer and the magnetization of all vortices cores, which get oriented along the $z$ direction during the domain formation process while 
(a)

(b)

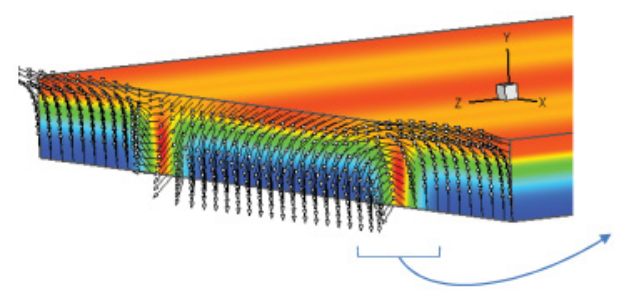

(c)
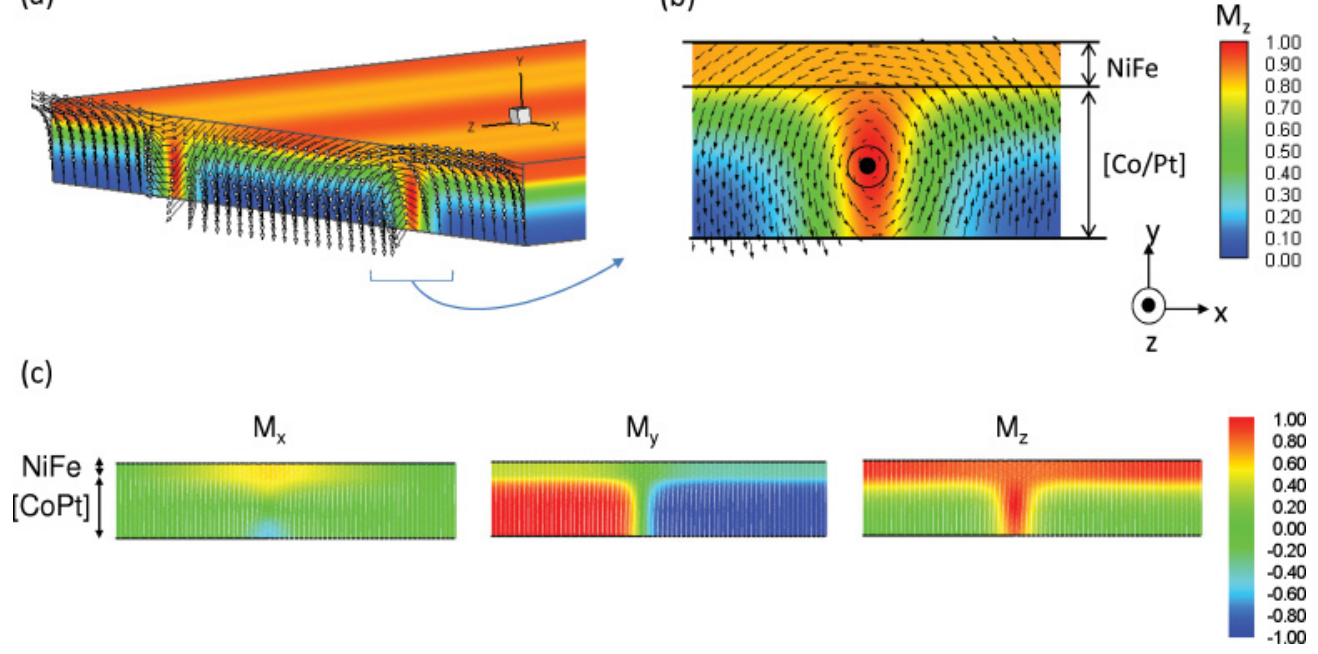

FIG. 8. (Color online) (a) 3D representation of the magnetization distribution simulated for $[\mathrm{Pt} / \mathrm{Co}] / \mathrm{NiFe}$ with $t_{\mathrm{Pt}}=25 \mathrm{~nm}$. The scale bar indicates the values for the magnetization component in the $z$ direction. (b) Zoom of the magnetization distribution close to a domain wall (cross-sectional view). The length of the arrows is proportional to the magnetization component in the plane of the figure. (c) Magnetization components $M_{x}, M_{y}$, and $M_{z}$ in a cross-sectional area. The color scale bar indicates the amplitudes of the magnetization components in the three axis directions.

decreasing the initial field from $z$ saturation to zero. This picture is consistent with the experimental observation that in the weak anisotropy systems $\left(t_{\mathrm{Pt}}=0\right.$ or $\left.1 \mathrm{~nm}\right)$, the domains are elongated along the initial in-plane field direction (Fig. 5). Indeed, in this case, the vortex core at the center of a given wall forms a line along the $z$ direction. From a magnetostatic point of view, the energy of the line is minimized if this line is straight along the $z$ direction. For larger anisotropy, it is likely that local pinning centers prevent the magnetic configurations from reaching a lower energy state, resulting in the observed labyrinth type of domain configurations. The fact that all the vortex cores are not aligned for thicker Pt buffers would tend to reduce the loop shift of the NiFe. Since this is not what is observed, we believe that this effect is negligible with respect to the loop shift enhancement resulting from the observed increased anisotropy for thicker Pt buffers (i.e. enhanced pinning strength of the vortices against magnetization reversal).

\section{CONCLUSIONS}

This paper shows the possibility of controlling the loop shift in a system consisting of two coupled ferromagnetic layers [Pt/Co]/NiFe having out-of-plane and easy-plane anisotropy by simply modifying the thickness of a Pt buffer layer, $t_{\mathrm{Pt}}$. A systematic study has allowed explaining the primary origin of the exchange-bias-like phenomenon observed in this system along the initial in-plane saturating field. This has been done by demonstrating the correlation existing between the structural quality of the [Pt/Co] films, the evolution of the corresponding out-of-plane anisotropy, $K_{u}$, and the variation of the magnetic domain structure at remanence after in-plane saturation when varying $t_{\mathrm{Pt}}$. The results showed three differentiated regions for the out-of-plane anisotropy of [Pt/Co] dependent on the Pt buffer thickness: (i) for $t_{\mathrm{Pt}}<5 \mathrm{~nm}$, the increase of $K_{u}$ with $t_{\mathrm{Pt}}$ is very pronounced due to a significant improvement in the multilayer structural quality; (ii) for values $5 \mathrm{~nm} \leqslant$ $t_{\mathrm{Pt}} \leqslant 20 \mathrm{~nm}$, a moderate increase of $K_{u}$ is observed; and (iii) for $t_{\mathrm{Pt}}>20 \mathrm{~nm}, K_{u}$ is approximately constant, no further structural improvement is observed. The magnetic structures measured by $\mathrm{MFM}$ for $[\mathrm{Pt} / \mathrm{Co}] / \mathrm{NiFe}$ and obtained at remanence after in-plane saturation of the system showed, according to the mentioned evolution, magnetic domains aligned parallel to the applied saturating field for systems with either no $\mathrm{Pt}$ buffer layer or an excessively thin layer $\left(t_{\mathrm{Pt}}<5 \mathrm{~nm}\right)$, whereas randomly oriented labyrinth magnetic domains were obtained with increasing $t_{\mathrm{Pt}}$.

Experimental results in combination with micromagnetic simulations have shown that the magnetic configuration responsible of the measured loop shift is the result of the exchange coupling between the magnetization of the $\mathrm{NiFe}$ layer and the magnetization of vortex cores (parallel to the applied field and all pointing towards the same direction), which form in the domain walls separating the upwards and downwards magnetized domains in the $[\mathrm{Pt} / \mathrm{Co}]$ multilayer. The formation of vortex cores after in-plane saturation of the $[\mathrm{Pt} / \mathrm{Co}] / \mathrm{NiFe}$ system marks the appearance of the exchange-bias phenomenon, and it is their resistance against magnetization reversal that dictates the magnitude of the loop shift obtained through a cycling with an in-plane magnetic field. The vortex pinning strength is given by the effective perpendicular anisotropy of the $[\mathrm{Pt} / \mathrm{Co}]$ multilayer, and this latter can be effectively enhanced by increasing the thickness of the Pt buffer layer.

\section{ACKNOWLEDGMENT}

A. Bollero acknowledges support through a Ramón y Cajal contract from the Spanish MICINN (formerly Spanish Ministry of Education and Science). 
*alberto.bollero@imdea.org

†vincent.baltz@cea.fr

${ }^{1}$ S. Tehrani, J. M. Slaughter, M. Deherrera, B. N. Engel, N. D. Rizzo, J. Slater, M. Durlam, R. W. Dave, J. Janesky, B. Butcher, K. Smith, and G. Grynkewich, Proc. IEEE 91, 703 (2003).

${ }^{2}$ For recent reviews, see J. Nogués and I. K. Schuller, J. Magn. Magn. Mater. 192, 203 (1999); A. E. Berkowitz and K. Takano, ibid. 200, 552 (1999); R. L. Stamps, J. Phys. D 33, R247 (2000); M. Kiwi, J. Magn. Magn. Mater. 234, 584 (2001).

${ }^{3}$ J. Camarero, Y. Pennec, J. Vogel, S. Pizzini, M. Cartier, F. Fettar, F. Ernult, A. Tagliaferri, N. B. Brookes, and B. Dieny, Phys. Rev. B 67, 020413(R) (2003).

${ }^{4}$ J. Nogués, J. Sort, S. Suriñach, J. S. Muñoz, M. D. Baro, J. F. Bobo, U. Lüders, E. Haanappel, M. R. Fitzsimmons, A. Hoffmann, and J. W. Cai, Appl. Phys. Lett. 82, 3044 (2003).

${ }^{5}$ J. Sort, A. Popa, B. Rodmacq, and B. Dieny, Phys. Rev. B 70, 174431 (2004).

${ }^{6}$ A. Bollero, L. D. Buda-Prejbeanu, V. Baltz, J. Sort, B. Rodmacq, and B. Dieny, Phys. Rev. B 73, 144407 (2006).

${ }^{7}$ W. C. Cain and M. H. Kryder, J. Appl. Phys. 67, 5722 (2006).

${ }^{8}$ P. Y. Yang, X. Y. Zhu, F. Zeng, and F. Pan, Appl. Phys. Lett. 95, 172512 (2009).

${ }^{9}$ J. Sort, K. S. Buchanan, V. Novosad, A. Hoffmann, G. SalazarAlvarez, A. Bollero, M. D. Baró, B. Dieny, and J. Nogués, Phys. Rev. Lett. 96, 067201 (2006).

${ }^{10}$ A. Bollero, B. Dieny, J. Sort, K. S. Buchanan, S. Landis, and J. Nogués, Appl. Phys. Lett. 92, 022508 (2008).
${ }^{11}$ C. L. Canedy, X. W. Li, and G. Xiao, J. Appl. Phys. 81, 5367 (1997).

${ }^{12}$ S. Zhang, Phys. Rev. B 51, 3632 (1995).

${ }^{13}$ B. Rodmacq, A. Manchon, C. Ducruet, S. Auffret, and B. Dieny, Phys. Rev. B 79, 024423 (2009).

${ }^{14}$ S. Landis, B. Rodmacq, and B. Dieny, Phys. Rev. B 62, 12271 (2000).

${ }^{15}$ V. Gehanno, Y. Samson, A. Marty, B. Gilles, and A. Chamberod, J. Magn. Magn. Mater. 172, 26 (1997).

${ }^{16}$ J. E. Davies, O. Hellwig, E. E. Fullerton, G. Denbeaux, J. B. Kortright, and K. Liu, Phys. Rev. B 70, 224434 (2004).

${ }^{17}$ S. Honda, Y. Ikegawa, and T. Kusuda, J. Magn. Magn. Mater. 111, 273 (1992)

${ }^{18}$ T. Kleinefeld, J. Valentin, and D. Weller, J. Magn. Magn. Mater. 148, 249 (1994).

${ }^{19}$ J. Valentin, T. Kleinefeld, and D. Weller, J. Phys. D 29, 1111 (1996).

${ }^{20}$ S. L. Tang, P. F. Carcia, D. Coulman, and A. J. McGhie, Appl. Phys. Lett. 59, 2898 (1991).

${ }^{21}$ I. Horcas, R. Fernández, J. M. Gómez-Rodríguez, J. Colchero, J. Gómez-Herrero, and A. M. Baro, Rev. Sci. Instrum. 78, 013705 (2007).

${ }^{22}$ U. Ebels, L. Buda, K. Ounadjela, and P. E. Wigen, Phys. Rev. B 63, 174437 (2001)

${ }^{23}$ S. Hashimoto, Y. Ochiai, and K. Aso, J. Appl. Phys. 66, 4909 (1989).

${ }^{24}$ S. Landis, B. Rodmacq, and B. Dieny, Phys. Rev. B 62, 12271 (2000). 